\title{
A variable-resolution optical profile measurement system
}

\author{
Tung-Hsien Tsai ${ }^{1}$, Kuang-Chao Fan ${ }^{2,4}$ and Jong-I Mou ${ }^{3}$ \\ ${ }^{1}$ Department of Mechanical Engineering, Hsiuping Institute of Technology, Taichung, \\ Taiwan, Republic of China \\ ${ }^{2}$ Department of Mechanical Engineering, National Taiwan University, Taipei, Taiwan, \\ Republic of China \\ ${ }^{3}$ Department of Industrial Engineering, Arizona State University, Tempe, Arizona, USA \\ E-mail: fan@ccms.ntu.edu.tw
}

Received 29 June 2001, in final form 30 November 2001, accepted for publication 5 December 2001

Published

Online at stacks.iop.org/MST/12

\begin{abstract}
In this study, a variable-resolution optical measurement system (VROPMS) based on triangulation measurement technology is proposed. The VROPMS optical scanning probe is composed of dual CCD cameras fitted with zoom lenses and a line laser diode projector. A flexible and novel calibration procedure for VROPMS is developed to acquire the system parameters quickly and accurately. The central position of the reflected laser image is calculated using Gaussian function least-squares fitting of the beam intensity. Subpixel resolution can thus be acquired. Experimental calibration results show that the higher the lens magnification, the finer the derived system resolution. The best accuracy at the zoomed focus position is about $0.02 \mathrm{~mm}$. This system can flexibly zoom in or out to measure a 3D object profile in sections according to the approximate surface profile. Varied mesh images taken from different zoom positions by VROPMS can be patched using the image matching technique to reconstruct the entire profile. A human sculpture with a complex surface profile is measured using VROPMS as a practical illustration of the effectiveness of the system.
\end{abstract} Ascii/Word/MST/mst126472/PAP Printed 17/12/2001 Issue no Total pages First page Last page File name Date req $(\mathrm{BJ})$

Keywords: optical profile measurement, variable mesh, 3D measurement (Some figures in this article are in colour only in the electronic version)

\section{Introduction}

Optical measurement systems using the triangulation principle to acquire the profile of 3D objects have been widely used. The most commonly used system projects a laser stripe onto the surface and uses a charge-coupled device (CCD) camera to capture the distorted line, and then converts it to a smooth spatial line using a curve fitting algorithm. By stepping the optical detector to prescribed positions, a series of deformed line images can be acquired. In this kind of system the resolution per pixel and the field of view are always fixed. In general, optical profile measurement systems have all been of the fixed focus type, mounted on a fixed three-axis stage for line

${ }^{4}$ Corresponding author. scanning [1-3]. Unless the system resolution can be changed flexibly, potential applications for this optical measurement system are restricted.

In this study, a variable resolution profile measurement system (VROPMS) based on scanning triangulation measurement technology [4] was developed. This new system provides variable focal lengths by adding a zoom lens to each of the conventional dual CCD camera systems. Theoretically, the higher the lens magnification, the finer the camera resolution that can be achieved. Using a zoom lens, the system's field of view can be further localized such that a larger curvature in a smaller area can be detected and a more approximate surface model can be reconstructed. In other words, this system can flexibly zoom in or out to measure a 3D object profile in sections according to the approximate requirements for a given surface 


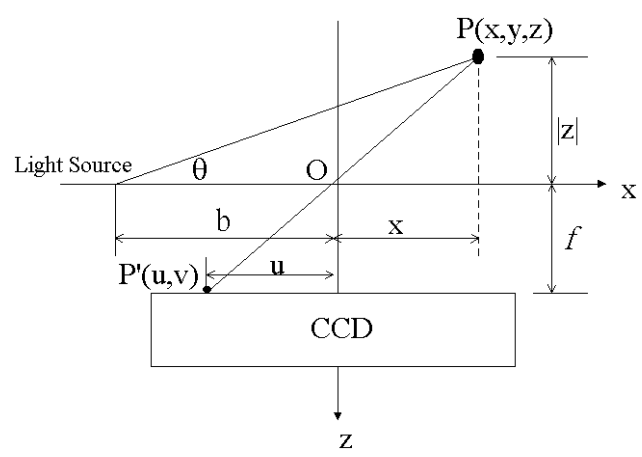

Figure 1. Principle of triangulation measurement.

profile. This research developed a software package so that the variable mesh images of an object acquired at various zoom positions can be matched using an image mapping technique to reconstruct the entire profile.

\section{Principle of triangulation measurement}

The principle of triangulation measurement with one camera is shown in figure 1 . In the figure, $P(x, y, z)$ is a point in the world coordinate $(X, Y, Z)$ and $P^{\prime}(u, v)$ is its focused point in the image plane $(U, V)$. According to geometrical optics and similar triangles, the coordinates of point $P(x, y, z)$ can be calculated using

$$
\begin{gathered}
\frac{f}{u}=\frac{|z|}{x} \\
\frac{b+x}{|z|}=\cot \theta
\end{gathered}
$$

where $f$ is the focal length, $\theta$ is the angle between the $X$-axis and light direction and $b$ is the distance between the light source and the lens optical centre $O$.

From equations (1) and (2), we can get

$$
x=\frac{b u}{f \cot \theta-u} .
$$

Similarly, in the $Y$-direction we have

$$
\frac{f}{v}=\frac{|z|}{y} \quad \text { and } \quad \frac{x}{y}=\frac{u}{v}
$$

which yields

$$
\begin{aligned}
& y=\frac{b v}{f \cot \theta-u} \\
& z=\frac{-b f}{f \cot \theta-u} .
\end{aligned}
$$

Therefore, the coordinates of point $P(x, y, z)$ are

$$
\left(\frac{b u}{f \cot \theta-u}, \frac{b v}{f \cot \theta-u}, \frac{-b f}{f \cot \theta-u}\right) .
$$

In principle, a system with one CCD camera can grab the projected line image on the surface and fit one free-form line at a time. Having scanned the entire image, an entire free-form surface profile of the object can be constructed. In practice, one CCD camera cannot always successfully capture the line image at each position because the inspected surface may have steep slopes and protruding profiles. To solve this problem, a

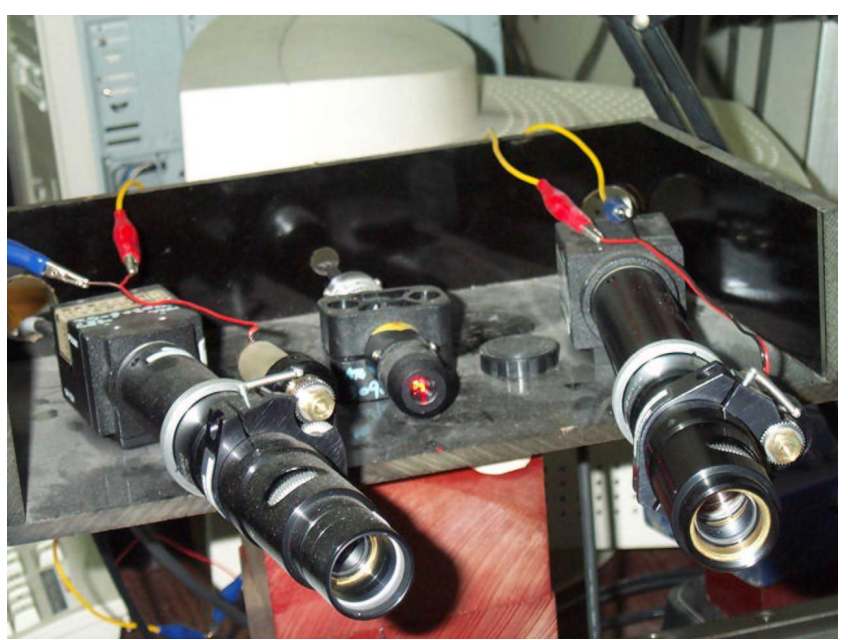

Figure 2. Scanning head of the VROPMS.

Table 1. Scanning head specifications of the VROPMS.

\begin{tabular}{lll}
\hline & Lowest & Highest \\
\hline Numerical aperture & 0.006 & 0.02 \\
Magnification & $0.071 \times$ & $0.50 \times$ \\
Field of view & $67.4 \mathrm{~mm} \times 89.8 \mathrm{~mm}$ & $9.5 \mathrm{~mm} \times 12.6 \mathrm{~mm}$ \\
Depth of field & $30 \mathrm{~mm}$ & $2.7 \mathrm{~mm}$ \\
Working distance & $350 \mathrm{~mm}$ & $350 \mathrm{~mm}$ \\
\hline
\end{tabular}

system with two CCD cameras is often adopted together with a line laser diode. Such a built-up optical head can scan over the object profile linearly or rotationally using the assisted moving stages.

\section{Principle and calibration method of the VROPMS}

\subsection{The VROPMS system}

In this study, the scanning head of the VROPMS is a laser diode line projector with two CCD cameras fitted with an OPTEM ZOOM 70 with a $0.38 \times$ TV tube and $0.25 \times$ auxiliary lens, as shown in figure 2. This optical scanning probe is mounted on a linear stage to execute the line-scanning measurement. The CCD camera has $768(\mathrm{H}) \times 494(\mathrm{~V})$ pixels and the laser diode is less than $5 \mathrm{~mW}$ with a wavelength of about $635 \mathrm{~nm}$. The framegrabber displays with a resolution of $480 \times 640$. The optical limits, such as the field of view and depth of field, are varied with different zooms. Table 1 lists the system specifications. The resolution of the original focus position is about $0.14 \mathrm{~mm} \mathrm{pixel}{ }^{-1}$ (67.4 mm, 480 pixel). The motorized zoom lens can be adjusted to any specified magnification. In this study, the magnification factors $(m)$ are controlled from 1 to 3 with incremental steps.

Figure 3 shows a schematic diagram of a $2 \mathrm{D}$ pinhole camera model, where $O^{\prime}$ is the projection centre and $R$ is the retinal plane. The optical axis is the line passing through the projection centre $O^{\prime}$ and perpendicular to the retinal plane. The intersection point of this line with the retinal plane is the principal point. The image position $A^{*}(U, V)$ of a space point $A(x, y, z)$ will be influenced by the camera parameters. There are two groups of parameters in a pinhole camera. The first group consists of intrinsic parameters and the second 


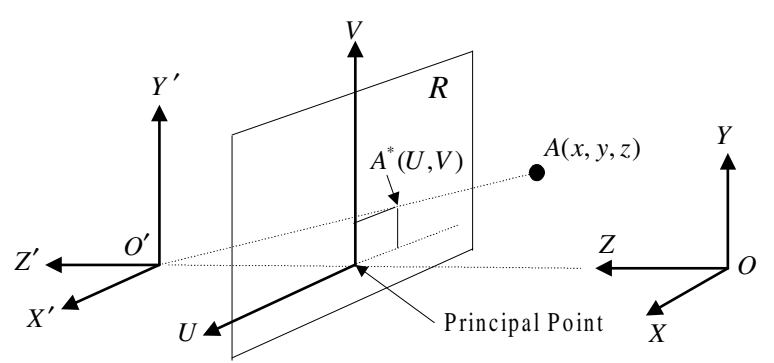

Figure 3. Schematic diagram of a 2D pinhole camera image model.

group contains extrinsic parameters. The extrinsic parameters characterize the position and orientation of the camera while the intrinsic parameters characterize the camera's projection properties. Dawson-Howe [4] and Rosenfield [5] presented the calibration procedure for the extrinsic parameters. Fryer [6], Curry [7], Ziemann [8], Hallert [9] and Brown [10] focused on the calibration of the lens distortion and the intrinsic parameters. In a zoom lens system, the extrinsic parameters are constant, but the intrinsic parameters will vary subject to the amount of zoom. Therefore, the VROPMS system parameters must be adjusted simultaneously with respect to the various zoom positions for exact computation of space coordinates.

The five intrinsic parameters are $P_{0}, Q_{0}, S_{u}, S_{v}$ and $\alpha\left(P_{0}, Q_{0}\right)$ indicates the coordinate of the principal point. $S_{u}$ and $S_{v}$ are the horizontal and vertical scale factors respectively, and $\alpha$ is the skew angle between the coordinate axes in the pixel coordinate system. Maybank [11] and Burner [12] observed that some of the intrinsic parameters were invariant with magnification, namely the aspect ratio $S_{u} / S_{v}$ and the skew angle $\alpha$. Therefore, there are only three variable intrinsic parameters when $m$ varies. Faugeras [13] found that calibrated parameters at different $m$, or zoom positions, could be modelled using the $S_{v}$ function. Sturm [14] proposed the following linear relationship between the intrinsic parameters: $S_{u}=1.466 S_{v} ; P_{0}=0.060 S_{v}+184.44 ; Q_{0}=-0.007 S_{v}+$ 273.19. According to this linear relationship, the idea of using the polynomial magnification fitting function factor, $m$, to find the transformation matrix between the image plane at magnification factor $m$ and the original focal plane $(m=1)$ was generated in this study [15]. A simple calibration method for acquiring the transformation function is also proposed. This calibration procedure can not only significantly reduce the calibration time, but can also flexibly acquire the system parameters at any focus position with adequate measurement accuracy. Based on this linear relationship, all of the intrinsic parameters can be mathematically fitted using only one variable, i.e., the magnification $m$. Details are described in the following sections.

\subsection{Determination of the laser beam centre}

The laser beam intensity normally presents a Gaussian distribution with $\mathrm{TEM}_{00}$ axial symmetry. The diameter is about 1-2 $\mathrm{mm}$. To acquire the centre position, general approaches in image processing are the zero crossing (ZC) method, the centroid method (CM) and the Fourier phaseshift (FPS) method. The ZC method is subject to ambient light disturbance. The CM method is sensitive to lowfrequency noises and is better for high-power lasers. The FPS

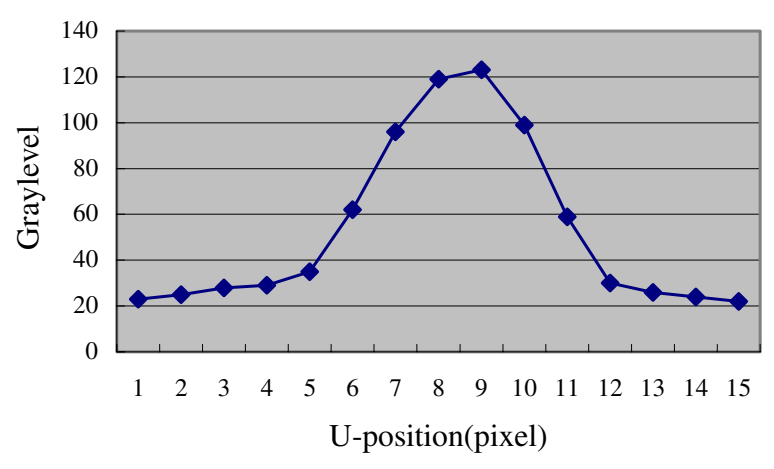

Figure 4. Measured laser beam intensity distribution along cross section.

method is more complex and requires larger system memory. This study proposes the smooth curve fitting (SCF) method to find the central position of the beam to the subpixel level.

An ideal laser line image captured by the CCD has a greylevel distribution along the cross sections in Gaussian form as

$$
I_{p}=B \exp \left(\frac{-(x-d)^{2}}{2 \sigma^{2}}\right)
$$

where $d$ indicates the beam centre position in the $X$ direction. In practice, the Gaussian curve will be slightly distorted with noise, as shown in figure 4 . It is not correct to calculate the $\mathrm{ZC}$ or centroid position directly from the captured image. In nature, the grey level must observe the Gaussian law. Therefore, a preliminary smoothing with a Gaussian curve can eliminate all disturbances caused by the optical and environmental components. Logarithmically, equation (6) can be written as

$$
\ln I_{p}=\ln B-\frac{(x-d)^{2}}{2 \sigma^{2}} .
$$

Let $y=\ln I_{p}, b=\ln B$ and $a=-1 / 2 \sigma^{2}$. Equation (7) can be rewritten as

$$
y=a(x-d)^{2}+b=a_{1} x^{2}+a_{2} x+a_{3} .
$$

This is a parabolic function with a peak point at $d$. Using this function to fit the measured grey-level data with the leastsquares method, the beam centre position $d$ can be computed to the subpixel level.

\subsection{Calibration procedures}

To acquire the exact space coordinates for different focus positions, the VROPMS system parameters must be adjusted simultaneously subject to the amount of zoom. The ordinary approach is to individually determine the system parameters with respect to each zoom position and enter this information in a lookup table. This calibration procedure is, however, timeconsuming and tedious during system setup. In addition, it provides only a discrete data bank suitable for certain particular zoom positions. Therefore, a flexible and novel calibration procedure for acquiring functional system parameters is proposed in this study. The required calibration procedures are divided into two steps. The objective of the first step is to convert the image coordinate from the magnification factor position, $m$, to its corresponding image coordinate at $m=1$ using the 'polynomial fitting functions'. The purpose of the 


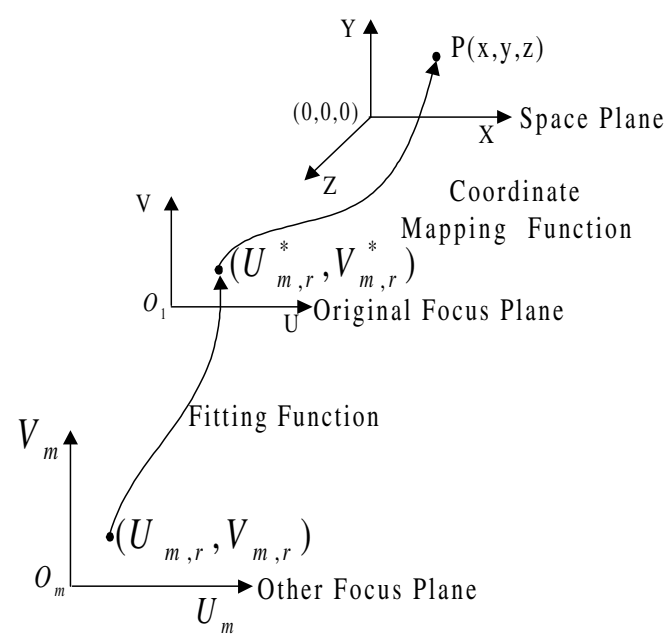

Figure 5. Schematic diagram for calibrating VROPMS.
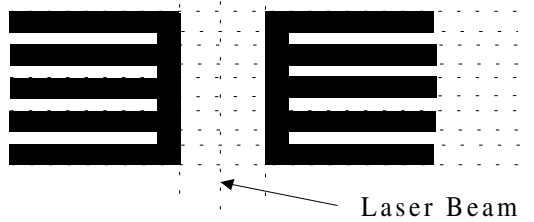

Figure 6. Template with $(10 \times 3)$ calibrating points.

second step is to use the 'coordinate mapping functions' to transform the 2D image data into corresponding 3D spatial positions at the original focal plane $(m=1)$. Figure 5 gives a schematic summary of the calibration procedures.

3.3.1. Calibration of the fitting function. Let $\left(U_{m}, V_{m}\right)$ be the image pixel coordinate at various zoom positions with magnification factors $m$. $\left(U_{m}^{*}, V_{m}^{*}\right)$ is the corresponding coordinate on the original focal plane $(m=1)$. The image correlation between the original focus position and the magnified focus position can be formulated by fitting the polynomial functions as $U_{m}^{*}=F_{U}\left(U_{m}, V_{m}, m\right)$ and $V_{m}^{*}=F_{V}\left(U_{m}, V_{m}, m\right)$. If the fitting functions are acquired, the image coordinate $\left(U_{m}^{*}, V_{m}^{*}\right)$ at the original focus position can then be calculated. The proposed calibration method is described below.

A laser stripe is projected onto the centre of a standard template that has 10 horizontal lines and two vertical lines generated by a laser writer with $1 \mu \mathrm{m}$ line spacing accuracy, as shown in figure 6. Each CCD camera captures the image and, by image processing and least-squares computation, calculates the intersection points between the three vertical dashed lines and the 10 horizontal dashed lines. This will create 30 standard points $\left(U_{r}, V_{r}\right)(r=0,1, \ldots, 29)$ at the original focus position.

Let the correspondence between coordinates $\left(U_{m}, V_{m}\right)$ and $\left(U_{m}^{*}, V_{m}^{*}\right)$ be a linear relationship with the following form:

$$
\begin{gathered}
U_{m}^{*}=f_{1}(m) \times U_{m}+f_{2}(m) \times V_{m}+f_{3}(m) \\
V_{m}^{*}=F_{1}(m) \times U_{m}+F_{2}(m) \times V_{m}+F_{3}(m)
\end{gathered}
$$

where $f_{i}$ and $F_{i}(i=1-3)$ are polynomial fitting functions of magnification factor $m$. Because as $m$ becomes larger, the field of view of the $\left(U_{m}, V_{m}\right)$ coordinates become smaller, it is reasonable to adopt a second-order inverse approximation to correlate $f_{i}(m)$ and $F_{i}(m)$. Equations (9) and (10) can be rewritten as

$$
\begin{aligned}
U_{m}^{*} & =\left[\left(A_{1} / m^{2}\right)+\left(A_{2} / m\right)+A_{3}\right] \times U_{m}+\left[\left(A_{4} / m^{2}\right)\right. \\
& \left.+\left(A_{5} / m\right)+A_{6}\right] \times V_{m}+\left[\left(A_{7} / m^{2}\right)+\left(A_{8} / m\right)+A_{9}\right] \\
V_{m}^{*} & =\left[\left(B_{1} / m^{2}\right)+\left(B_{2} / m\right)+B_{3}\right] \times U_{m}+\left[\left(B_{4} / m^{2}\right)\right. \\
& \left.+\left(B_{5} / m\right)+B_{6}\right] \times V_{m}+\left[\left(B_{7} / m^{2}\right)+\left(B_{8} / m\right)+B_{9}\right]
\end{aligned}
$$

where $A_{i}$ and $B_{i}(i=1,2, \ldots, 9)$ are polynomial coefficients of $f_{i}(m)$ and $F_{i}(m)$.

For computing $A_{i}$ and $B_{i}$, the focus position is changed sequentially and the magnification $m$ is varied from 1 to 3 in seven designated steps $(m=$ 1.0, 1.25, 1.5 1.75, 2.0, 2.5, 3.0). Each designated focus position has its own intersection points $\left(U_{m, r}, V_{m, r}\right), 10$ points on each reference line for three lines. Thus, the approximated linear fitting functions for each intersection point are expressed as follows:

$$
\begin{aligned}
U_{m, r}^{*} & =\sum_{i=1}^{3}\left(A_{i} / m^{3-i}\right) U_{m, r}+\sum_{j=4}^{6}\left(A_{j} / m^{6-j}\right) U_{m, r} \\
& +\sum_{k=7}^{9} A_{k} / m^{9-k} \\
V_{m, r}^{*} & =\sum_{i=1}^{3}\left(B_{i} / m^{3-i}\right) U_{m, r}+\sum_{j=4}^{6}\left(B_{j} / m^{6-j}\right) U_{m, r} \\
& +\sum_{k=7}^{9} B_{k} / m^{9-k} .
\end{aligned}
$$

The residuals $E_{u}$ and $E_{v}$ of $U_{m, r}^{*}$ and $V_{m, r}^{*}$ respectively can be derived as $E_{u}=\sum_{r=0}^{29} \sum_{m=1}^{N}\left(U_{m, r}^{*}-U_{r}\right)^{2}$ and $E_{v}=$ $\sum_{r=0}^{29} \sum_{m=1}^{N}\left(V_{m, r}^{*}-V_{r}\right)^{2}$. The coefficients $A_{i}$ and $B_{i}$ can be determined using least squares approximation: $\partial E_{u} / \partial A_{i}=0$ and $\partial E_{v} / \partial B_{i}=0$. Therefore, the related image coordinates $\left(U_{m, r}^{*}, V_{m, r}^{*}\right)$ for any focus position that has magnification $m$ can be obtained.

3.3.2. Calibration of coordinate mapping function. The calibration principle for the coordinate mapping functions is shown in figure 7. A laser stripe is projected onto a standard template design, and the CCD cameras detect the line image. Because the distance between each horizontal line on the standard template is known, the intersection point between each horizontal line and the vertical laser line stripe indicates a calibration point on the template. Since the template in use is designed with 15 horizontal lines, the total number of calibration points is 15 . In the experiment, the template was successively moved step-by-step along the $Z$-axis direction to prescribed positions. At each $Z$-position, each CCD camera captures the image and calculates the coordinates of all calibration points. From the structural geometry in figure 7, it can be seen that the $X$-coordinate component of a position in space is constant throughout the scanned lines along the $Y-Z$ plane. Thus, only two space coordinate components $(Y, Z)$ need to be transformed from the data on the image plane using the least-squares mapping algorithm.

Let $\left(Y_{k}, Z_{k}\right)$ be a coordinate of the standard template in the space plane, and $\left(U_{k}^{*}, V_{k}^{*}\right)$ the corresponding image coordinate 
Table 2. The average fitting errors of corresponded image coordinates (unit: pixel).

\begin{tabular}{|c|c|c|c|c|c|c|c|}
\hline \multicolumn{4}{|c|}{$U$} & \multicolumn{4}{|c|}{$V$} \\
\hline Number & Error & Number & Error & Number & Error & Number & Error \\
\hline 1 & 0.45 & 6 & 0.45 & 1 & 1.06 & 6 & 1.05 \\
\hline 2 & 0.45 & 7 & 0.44 & 2 & 1.04 & 7 & 1.05 \\
\hline 3 & 0.46 & 8 & 0.46 & 3 & 1.00 & 8 & 1.05 \\
\hline 4 & 0.46 & 9 & 0.46 & 4 & 1.06 & 9 & 1.06 \\
\hline 5 & 0.46 & 10 & 0.46 & 5 & 1.11 & 10 & 1.02 \\
\hline Average & 0.45 & & & Average & 1.05 & & \\
\hline $2 \sigma$ & 0.01 & & & $2 \sigma$ & 0.03 & & \\
\hline
\end{tabular}

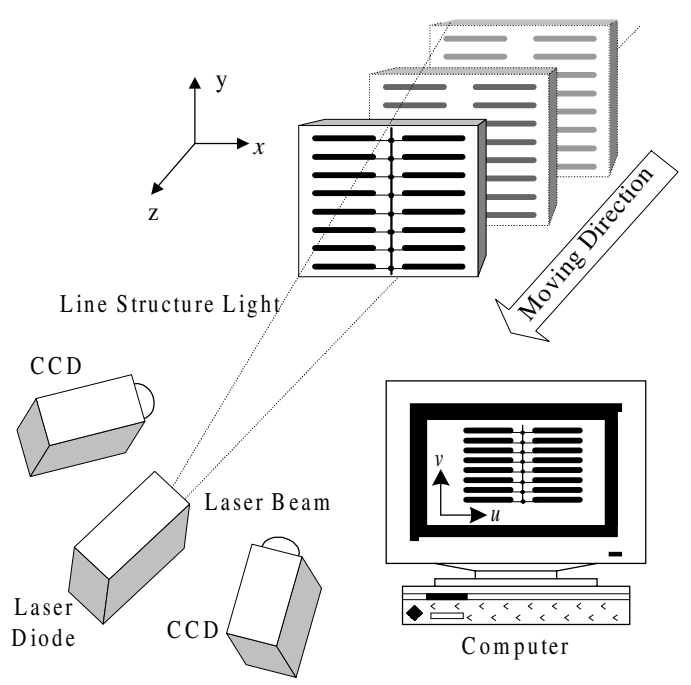

Figure 7. Calibration principle for coordinate mapping function .

in the original focus plane. A mapping algorithm can then be established using the least-squares polynomial function as follows:

$$
\begin{aligned}
& Y\left(U_{k}^{*}, V_{k}^{*}\right)=\sum_{j=0}^{M} \sum_{i=0}^{M-j} C_{Y_{i j}} U_{k}^{* i} V_{k}^{* j} \\
& Z\left(U_{k}^{*}, V_{k}^{*}\right)=\sum_{j=0}^{M} \sum_{i=0}^{M-j} C_{Z_{i j}} U_{k}^{* i} V_{k}^{* j}
\end{aligned}
$$

where $C_{y_{i j}}$ and $C_{z_{i j}}$ are the coefficients of the polynomial mapping functions, and $M$ is the order of the approximated polynomial function.

The residual error functions $E_{y}$ and $E_{z}$ of $Y\left(U_{k}^{*}, V_{k}^{*}\right)$ and $Z\left(U_{k}^{*}, V_{k}^{*}\right)$ respectively can be obtained using

$$
E_{Y}=\sum_{k=0}^{N}\left(Y_{k}-Y\right)^{2} \quad E_{Z}=\sum_{k=0}^{N}\left(Z_{k}-Z\right)^{2}
$$

where $N$ is the total number of calibration points.

The coefficients $C_{y_{i j}}$ and $C_{z_{i j}}$ can be determined from the minimum error.

$$
\frac{\partial E_{Y}}{\partial C_{Y_{i j}}}=0 \quad \frac{\partial E_{Z}}{\partial C_{Z_{i j}}}=0
$$

From equations (15) and (16), the corresponding space coordinate $(Y, Z)$ to each pixel position $(U, V)$ of the laser beam image on each of the CCD planes can be determined. Hence, with an $X$-position controlled line scanning system, combining the determined $Y$ and $Z$ components with the constant $X$ component on each scanned line, the entire profile can be formed by integrating the total measured data.

In summary, the VROPMS needs two data conversion steps. In the first step, the image coordinates $\left(U_{m}, V_{m}\right)$ for the focus position at magnification $m$ can be detected using CCD cameras. Substituting $\left(U_{m}, V_{m}\right)$ into the fitting functions of equations (13) and (14), the corresponding image coordinates $\left(U_{m}^{*}, V_{m}^{*}\right)$ can be calculated. In the second step, the corresponding space coordinates $(Y, Z)$ can be derived from the image coordinates $\left(U_{m}^{*}, V_{m}^{*}\right)$ using the coordinate mapping functions from equations (15) and (16). Lastly, combining the measured $Y$ and $Z$ components of each profile line from the image information with the positioned $X$ component from the scanning stage, the entire profile can be formed with respect to the focus position at magnification $m$.

\section{Calibrated errors of VROPMS}

\subsection{Fitting function error analysis}

In the experiment, the fitting function calibration procedure was executed repeatedly 10 times. For each calibration procedure, the VROPMS magnification was adjusted by assigning $m=1.0,1.25,1.5,1.75,2.0,2.5$ and 3.0 sequentially to obtain the image coordinates of each standard calibration point. The coefficients $A_{i}$ and $B_{i}$ were computed and the corresponding image coordinates $\left(U_{m}^{*}, V_{m}^{*}\right)$ for each focus position were determined. The average fitting errors $\left(E_{u}, E_{v}\right)$ of the related image coordinates were compared with the original image coordinates in each calibration, as shown in table 2.

Table 2 shows that the fitting errors are approximately 0.45 and 1.05 pixels in the $U$ and $V$ directions respectively. The repeatability is about $0.01-0.03$ pixels with $95 \%$ confidence level. It was found that larger fitting errors appeared on the boundary points. This is probably caused by the lens distortion. Therefore, the object should be placed as close to the centre of the image as possible to increase the measurement accuracy of the system.

In addition to the linear function approach, a nonlinear function was also attempted to derive the fitting function. The results, however, were no better than the linear case. Thus, the assumption of a linear relationship of the intrinsic parameters is satisfactory.

\subsection{Mapping function error analysis}

In this experiment (see figure 7), the calibration template was moved step by step to 20 positions at $1.5 \mathrm{~mm}$ intervals along the 
Table 3. The absolute average error of mapping function (unit: $\mathrm{mm}$ ).

\begin{tabular}{lllllllll}
\hline & \multicolumn{3}{c}{$Z$} & & \multicolumn{5}{c}{$Y$} \\
\cline { 8 - 9 } \cline { 6 - 8 } Number & Error & Number & Error & & Number & Error & Number & Error \\
\hline 1 & 0.092 & 6 & 0.090 & & 1 & 0.036 & 6 & 0.036 \\
2 & 0.090 & 7 & 0.094 & & 2 & 0.039 & 7 & 0.038 \\
3 & 0.086 & 8 & 0.094 & 3 & 0.043 & 8 & 0.038 \\
4 & 0.093 & 9 & 0.091 & & 0.041 & 9 & 0.041 \\
5 & 0.086 & 10 & 0.094 & 5 & 0.037 & 10 & 0.040 \\
Average & 0.091 & & & Average & 0.039 & & \\
$2 \sigma$ & 0.003 & & & $2 \sigma$ & 0.002 & & \\
\hline
\end{tabular}

Table 4. Average dimension measurement results with various magnifications.

\begin{tabular}{lll}
\hline$m$ & Result $(\mathrm{mm})$ & \multicolumn{1}{c}{ Error $(\mathrm{mm})$} \\
\hline 1.0 & 18.0872 & 0.0667 \\
1.25 & 17.9687 & -0.0523 \\
1.5 & 17.9715 & -0.0495 \\
1.75 & 17.9830 & -0.0380 \\
2.0 & 18.0560 & 0.0350 \\
2.5 & 18.0481 & 0.0271 \\
3.0 & 17.9965 & -0.0245 \\
\hline
\end{tabular}

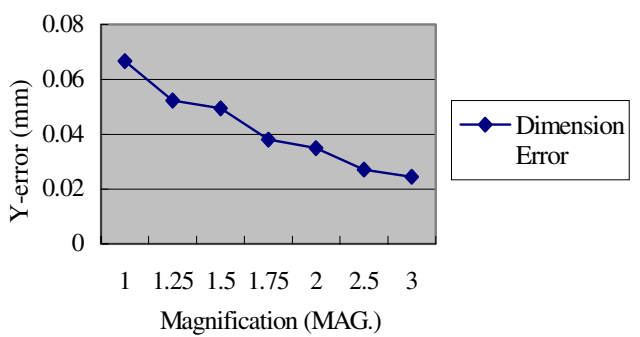

Figure 8. The dimension accuracy with various magnifications.

$Z$-axis. The coefficients of the coordinate mapping functions were determined using the minimum error functions with equations (12)-(15). The calibration procedure was executed repeatedly 10 times using the same fitting function parameters. Each time the corresponding coordinates were calculated with respect to the 15 calibration points. The absolute average mapping errors of the corresponding coordinates to the original real coordinates are shown in table 3 .

The calibrated errors are approximately 0.091 and $0.039 \mathrm{~mm}$ in the $Z$ - and $Y$-directions, respectively. The repeatability is about $0.002-0.003 \mathrm{~mm}$ with $95 \%$ confidence. Similar to the fitting function calibration, larger errors were also found at the outer points.

\subsection{System measurement errors}

Standard objects were measured in the $Y$-axis and $Z$-axis direction respectively to examine the in-plane dimension accuracy and depth accuracy of the developed VROPMS.

4.3.1. In-plane dimension accuracy. A template with a known etching length of $18.021 \mathrm{~mm}$ was fixed to examine the in-plane measurement accuracy. The system magnification was adjusted from 1.0 to 3.0 with a designated magnification ( $m=1.0,1.25,1.5,1.75,2.0,2.5,3.0)$ and the template was measured 10 times. The average measurement result for
Table 5. Average depth measurement results of various magnifications.

\begin{tabular}{lll}
\hline$m$ & Results $(\mathrm{mm})$ & Error $(\mathrm{mm})$ \\
\hline 1.0 & 1.9624 & -0.0926 \\
1.25 & 1.9810 & -0.0740 \\
1.5 & 1.9861 & -0.0689 \\
1.75 & 2.0058 & -0.0492 \\
2.0 & 2.0195 & -0.0355 \\
2.5 & 2.0201 & -0.0349 \\
3.0 & 2.0221 & -0.0329 \\
\hline
\end{tabular}

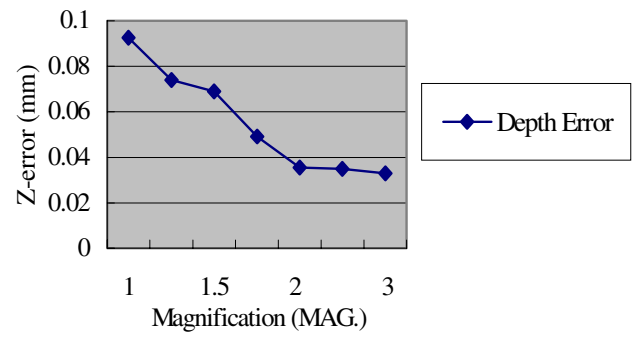

Figure 9. The depth accuracy with various magnifications.

each magnification is shown in table 4. The dimension accuracy of various magnifications is illustrated in figure 8 . The results show that dimension accuracy increases with increased magnification and the measurement error for each magnification is less than the corresponding theoretical camera resolution $(0.14 \mathrm{~mm})$. Hence, for a complex profile object that has a larger area of curvature, the system magnification can be flexibly adjusted to carry out the local finer resolution measurement for acquiring a more approximate surface model.

4.3.2. Depth accuracy. A plate with a thickness of $2.055 \mathrm{~mm}$ was used to examine the depth accuracy. The plate was measured 10 times repeatedly with various magnifications ( $m=1.0,1.25,1.5,1.75,2.0,2.5,3.0)$. The average measurement results for each magnification are shown in table 5. The tendency of the depth accuracy of various magnifications is shown in figure 9. The depth measurement error for each magnification is also smaller than the corresponding theoretical camera resolution. Because the horizontal image coordinates depend on the variation of depth, the detected central laser position will dramatically influence the depth accuracy. Although least-squares computation was used, the detected laser centre position can be improved to subpixel resolution, but the contribution is less than the result in the $Y$-axis direction. 


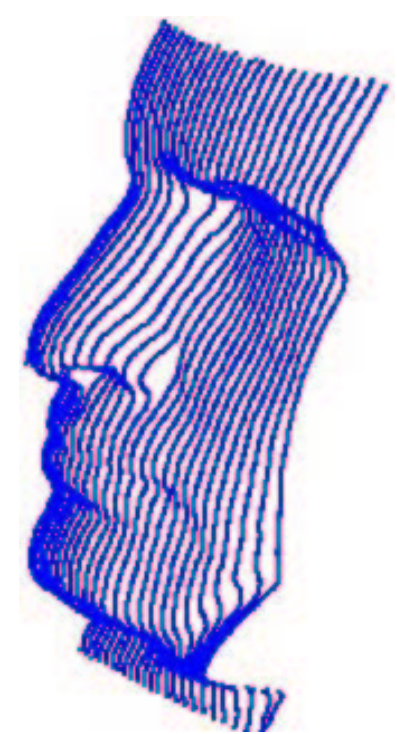

Figure 10. The measurement data points of a human sculpture using VROPMS linear scanning.

The results of the experimental measurement show that the system accuracy is about 0.07 and $0.09 \mathrm{~mm}$ in the $Y$ axis and $Z$-axis directions respectively at the original focus position. The system accuracy is approximately $0.03 \mathrm{~mm}$ when the magnification $m$ equals 3 . Thus, the higher the system magnification is, the finer the resolution that can be achieved.

\section{Applications}

A complex profile generally has a complicated curvature distribution, such as the nose area of a human head sculpture. In a larger curved area, the number of measured points must be large enough to reconstruct an approximate surface model. In other words, more measured points and a finer resolution are required to describe a larger curved area. In practice, the object was measured initially using VROPMS with a large scanning interval using the original focus position. The slope distribution of initial measured data can then be computed. According to the result of the slope analysis, the larger curved area can be identified and the zoom lens is adjusted to a higher magnification considering the depth of field and optical limits of the field of view to measure the identified area with a smaller scanning interval. A series of sectional measurement images that have different resolution meshes can therefore be acquired. Through registering sectional measurement images using an image-matching algorithm, an entire object profile that has several different resolution areas can be integrated.

Figure 10 illustrates the measurement data points taken using the VROPMS linear scanning measurement system [16] with a pitch of $1 \mathrm{~mm}$ in the original focus position. The slope distribution of the scanning profile is shown in figure 11. Both figures 10 and 11 show that the ambient area near the nose has a larger curved surface. In this case, the local steeper area is about $5 \mathrm{~mm} \times 15 \mathrm{~mm}$, and the variation in the depth of the object is about $10 \mathrm{~mm}$. The resolution in this area is not satisfactory. The system magnification was adjusted to 2 and the nose area was scanned again with a pitch of $0.5 \mathrm{~mm}$ to form a finer localized measurement, as shown in figure 12. There are 2142 points in the area of the lapping image. Consequently,
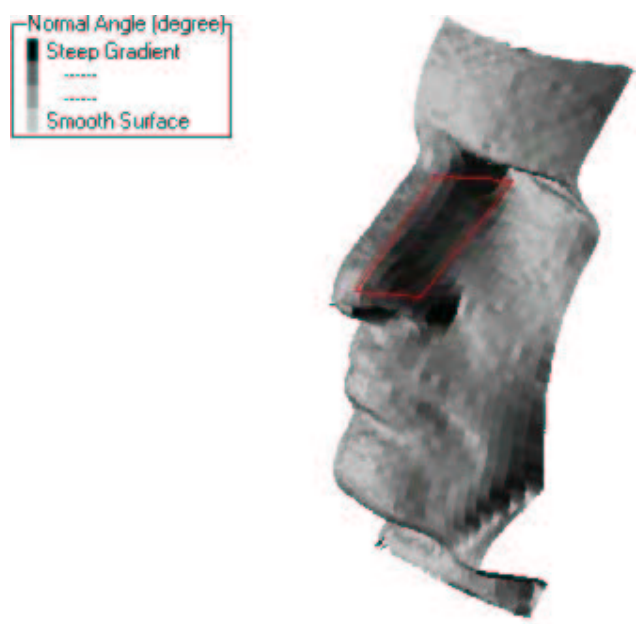

Figure 11. The slope distribution of a human sculpture.

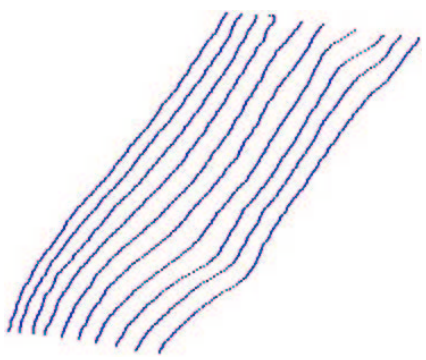

Figure 12. Local measurement data points for the nose of a human sculpture.

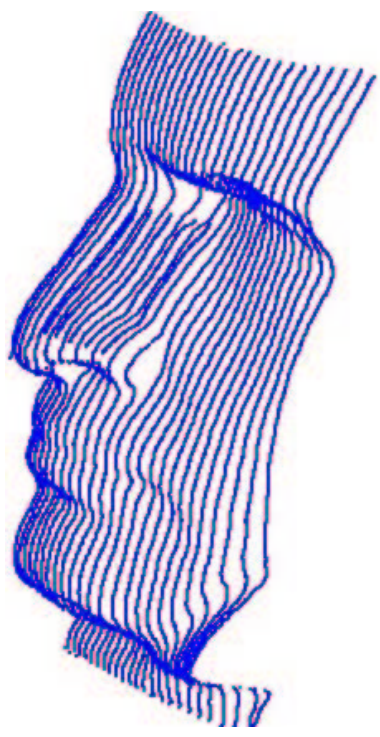

Figure 13. The merged data points of the human sculpture.

the localized measurement data must be stitched to the original macro data. This principle is similar to the CAE analysis of variable meshes for constructing an entire profile. Two various mesh images are registered optimally using the optimum shape error analysis [16]. The number of measured data points for this sculpture with different meshes is shown in figure 13. The results show that the approximate surface model for a larger curved area can be made more lifelike using VROPMS and an optimal image matching method. 


\section{Conclusions}

A flexible and novel variable resolution optical profile measurement system and its application were proposed in this study. The related image fitting function between the original focus position and another focus position can be acquired using the fitting function calibration procedure. The coordinate mapping function calibration procedure can then be used to obtain the space coordinate mapping function between the space plane and the image plane. This combined calibration procedure is quick and easy to use with promising system accuracy. In practice, the total time for system calibration is less than $10 \mathrm{~min}$.

For measuring a complex 3D object, the VROPMS can flexibly zoom in or out to measure an object profile according to its slope distribution or the requirements of profile approximation. The higher the adopted lens magnification, the finer and more accurate the surface profile that can be acquired. With the proposed fine and rough image matching algorithm, the developed VROPMS provides better flexibility and is more accurate for non-contact measurement.

\section{References}

[1] Aguilar J J, Torres F and Lope M A 1996 Stereo vision for 3D measurement accuracy analysis, calibration and industrial applications Measurement 18 193-200

[2] Ho C C 1997 3D Surface matching techniques in image measurements Masters Thesis National Taiwan University
[3] Tai W C and Chang M 1996 Non-contact profilometric measurement of large-form parts Opt. Eng. 35 $2730-27353$

[4] Dawson-Howe K M and Vernon D 1994 Simple pinhole camera calibration Int. J. Imaging Syst. Technol. 5 1-6

[5] Rosenfield G H 1970 The problem of exterior orientation in photogeometry Photogeom. Eng. 36 584-8

[6] Fryer J G and Brown D C 1986 Lens distortion for close-range photogeometry Photogeom. Eng. Remote Sens. 52 51-8

[7] Curry S and Baumrind S 1986 Calibration of array camera Photogeom. Eng. Remote Sens. 52 627-36

[8] Ziemann H and El-Hakim S F 1986 System calibration and self-calibration: Part I. Rotationally symmetrical lens distortion and image deformation Photogeom. Eng. Remote Sens. 52 1617-25

[9] Hallert B 1966 Quality of exterior orientation Photogeom. Eng. 32 464-75

[10] Brown D C 1971 Close-range camera calibration Photogeom. Eng. 37 855-66

[11] Maybank S J and Faugeras O D 1992 A theory of self calibration of a moving camera Int. J. Comput. Vis. 8 123-51

[12] Burner A W 1995 Zoom lens calibration for wind tunnel measurements Proc. SPIE 2598 19-33

[13] Faugeras O D and Toscani G 1987 Camera calibration for 3D computer vision Proc. Int. Workshop Machine Vision and Machine Intelligence (Tokyo)

[14] Sturm P 1997 Self-calibration of a moving zoom-lens camera by pre-calibration Image Vis. Comput. 15 583-9

[15] Tsai T H and Fan K C 2000 A mathematical model and procedure for calibrating a 3D measurement system with a zoom-lens camera J. Chin. Soc. Mech. Eng. 21 223-7

[16] Fan K C and Tsai T H 2001 Optimal shape error analysis of the matching image for a free-form surface Robot. Comput.-Integr. Manuf. 17 215-22 


\section{Queries for IOP paper 126472}

\section{Journal: $\quad$ MST}

Author: $\quad$ T-H Tsai et al

Short title: A variable-resolution optical profile measure-

\section{ment system}

\section{Page 1}

Query 1:

Author: please be aware that any colour figures in this article will only be printed in colour if you have previously arranged this with the Production Editor.

\section{Page 8}

Query 2:

Author: Please check page number of [3].

\section{Query 3:}

Author: Publisher and page numbers for reference [13]? 